\title{
Measuring physical activity in older adults: calibrating cut-points for the MotionWatch $8^{\odot}$
}

\author{
Glenn J. Landry',2t, Ryan S. Falck ${ }^{1,2+}$, Michael W. Beets ${ }^{3}$ and Teresa Liu-Ambrose ${ }^{1,2,4 *}$ \\ ${ }^{1}$ Aging, Mobility, and Cognitive Neuroscience Lab, Department of Physical Therapy, Faculty of Medicine, University of British \\ Columbia, Vancouver, BC, Canada, ${ }^{2}$ Djavad Mowafaghian Centre for Brain Health, University of British Columbia, \\ Vancouver, BC, Canada, ${ }^{3}$ Department of Exercise Science, Arnold School of Public Health, University of South Carolina, \\ Columbia, SC, USA, ${ }^{4}$ Brain Research Centre, University of British Columbia, Vancouver, BC, Canada
}

\section{OPEN ACCESS}

Edited by:

P. Hemachandra Reddy,

Texas Tech University, USA

Reviewed by:

Ramesh Kandimalla,

Texas Tech University, USA Annette N. Boles,

Texas Tech University Health

Sciences Center, USA

*Correspondence:

Teresa Liu-Ambrose,

Aging, Mobility, and Cognitive Neuroscience Lab, Department

of Physical Therapy, Faculty of Medicine, University of British Columbia, 212 - 2177 Wesbrook Mall, Vancouver, BC V6T 1Z3,

Canada

teresa.ambrose@ubc.ca

†Shared first authorship

Received: 13 April 2015 Accepted: 11 August 2015

Published: 25 August 2015

Citation:

Landry GJ, Falck RS, Beets MW and Liu-Ambrose T (2015) Measuring physical activity in older adults: calibrating cut-points

for the MotionWatch $8^{\odot}$.

Front. Aging Neurosci. 7:165. doi: 10.3389/fnagi.2015.00165
Given the world's aging population, the staggering economic impact of dementia, the lack of effective treatments, and the fact a cure for dementia is likely many years away - there is an urgent need to develop interventions to prevent or at least delay dementia's progression. Thus, lifestyle approaches to promote healthy aging are an important line of scientific inquiry. Good sleep quality and physical activity (PA) are pillars of healthy aging, and as such, are an increasing focus for intervention studies aimed at promoting health and cognitive function in older adults. However, PA and sleep quality are difficult constructs to evaluate empirically. Wrist-worn actigraphy (WWA) is currently accepted as a valid objective measure of sleep quality. The MotionWatch $8^{\odot}$ (MW8) is the latest WWA, replacing the discontinued Actiwatch 4 and Actiwatch 7. In the current study, concurrent measurement of WWA and indirect calorimetry was performed during 10 different activities of daily living for 23 healthy older adults (aged 57-80 years) to determine cut-points for sedentary and moderate-vigorous PA - using receiver operating characteristic curves - with the cut-point for light activity being the boundaries between sedentary and moderate to vigorous PA. In addition, simultaneous multi-unit reliability was determined for the MW8 using inter-class correlations. The current study is the first to validate MW8 activity count cut-points - for sedentary, light, and moderate to vigorous PA - specifically for use with healthy older adults. These cut-points provide important context for better interpretation of MW8 activity counts, and a greater understanding of what these counts mean in terms of PA. Hence, our results validate another level of analysis for researchers using the MW8 in studies aiming to examine PA and sleep quality concurrently in older adults.

Keywords: accelerometers, actigraphy, indirect calorimeters, validation, physical activity, older adults

\section{Introduction}

Worldwide, one new case of dementia is detected every $4 \mathrm{~s}$ (WHO and ADI, 2012). Given the world's aging population, the staggering economic impact of dementia (Wimo and Prince, 2010), the lack of effective treatments, and the fact a cure for dementia is likely many years away - there is an urgent need to develop interventions to prevent or at least delay dementia's progression (for review, see Landry and Liu-Ambrose, 2014). Thus, lifestyle approaches to promote healthy aging have become an important line of scientific inquiry. 
Good quality sleep is a pillar of healthy aging. Unfortunately, sleep changes as a function of normal aging, both in terms of decreased quality and quantity (for reviews, see Espiritu, 2008; Crowley, 2011). Sleep complaints are common in older adults more than half of adults $65+$ have at least one chronic sleep complaint - the most common being an inability to stay asleep at night (Foley et al., 1995). Of critical importance is the growing evidence suggesting poor sleep quality is a contributing factor in the prevalence, progression, and severity of Alzheimer's disease the most common cause of dementia (Landry and Liu-Ambrose, 2014).

Physical activity (PA) is another important pillar of healthy aging. Epidemiological evidence suggests PA increases life span and reduces the risk of chronic conditions such as stroke, heart disease, dementia, and type-2 diabetes (Blair and Brodney, 1999; Nelson et al., 2007; Blair, 2009; Chodzko-Zajko et al., 2009; Hamer and Chida, 2009; Ahlskog et al., 2011; Erickson et al., 2012; Swift et al., 2013). Furthermore, PA in the form of exercise has long been thought to play an important role in the quality of sleep. Epidemiological studies examining the relationship between exercise and sleep (for reviews, see Driver and Taylor, 2000; Youngstedt, 2005) consistently show that: (1) when asked an open ended question about behaviors that promote better sleep, exercise is listed as the most important; and (2) based on selfreport data, people who exercise more also report having better quality sleep and reduced daytime sleepiness - when compared with individuals who are more sedentary. Exercise effects on sleep quality might be explained by the chronobiotic properties of exercise (i.e., the ability to synchronize circadian rhythms of physiology and behavior). The chronobiotic properties of exercise have been well established in various animal models (for reviews, see Mrosovsky, 1996; Hastings et al., 1998), however, the science is less definitive in humans (reviewed in Mistlberger and Skene, 2004, 2005).

As such, recent research efforts have increased focus on PA and improved circadian regulation of sleep-wake rhythms as means to promote health and cognitive function in older adults (for relevant reviews, see Colcombe and Kramer, 2003; Landry and Liu-Ambrose, 2014). However, PA and sleep quality are complex constructs to evaluate empirically. Thus, we suggest the validity of future research efforts depends greatly on the methods used to measure parameters of PA and sleep quality.

Recent technological advances have led to the development of battery powered, long-life, light-weight, non-invasive, wristworn accelerometers that measure tri-axial movement (i.e., actigraphy). Wrist-worn accelerometers (WWAs) have been validated for measurement of sleep parameters by comparison with polysomnography (PSG; the gold standard objective measure of sleep; Littner et al., 2003a); and thus, actigraphy is currently accepted as a valid, practical alternative to PSG, allowing for long-term continuous sleep assessments at home (Ancoli-Israel et al., 2003; Littner et al., 2003b). Importantly, objective measures (i.e., actigraphy using WWA) survey different aspects of sleep quality - when compared with commonly used subjective measures such as the Pittsburgh Sleep Quality Index (PSQI; Buysse et al., 1989) and the Consensus Sleep Diary (CSD; Carney et al., 2012).
In a companion study to the current study, we provided evidence showing that PSQI and CSD reported sleep quality yields little to no predictive validity for actigraphic measures of sleep quality for older adults (Landry et al., 2015). We compared subjective (i.e., the PSQI and CSD) and objective measures (i.e., actigraphy using WWA) of sleep quality. For actigraphic measurement of sleep quality, we used the latest WWA the MotionWatch $8^{\complement}$ actigraphy system (MW8; CamNtech, Cambridge, UK), which replaced the discontinued Actiwatch 4 and Actiwatch 7. The companion study's findings suggested that perceived sleep quality is quite different from objective reality, at least for older adults. We believe these results to be important for researchers aiming to include sleep quality as an outcome measure in their studies. As such, we expect actigraphy will be used more widely in future studies examining PA and sleep quality among older adults.

As with sleep quality, precise measurement of PA is critical to advancing our understanding of PA in both health and disease. The general consensus is that objective measures of PA provide more precise measurements in older adult populations (Warren et al., 2010; Kowalski et al., 2012; Falck et al., 2015). In addition to its utility as an objective measure of sleep quality, the MW8 provides long-term continuous 24/7 recordings of PA. As such, the MW8 is an attractive alternative to previously used measures of PA behavior in older adults. The MW8 provides distinct advantages over other objective measures of PA, such as pedometers and hip-worn accelerometers. First, the MW8 simultaneously provides long-term continuous measures of sleep quality and PA. Second, unlike pedometers and hip-worn accelerometers, the MW8 is waterproof and does not need to be removed during activities such as bathing or swimming. Third, the MW8 is specifically designed for comfortable, continuous wear during the day and at night, which improves wearer compliance and minimizes missing data. Finally, the MW8 might capture light activities better (i.e., household chores), especially those that do not involve the lower extremities.

Clearly, the MW8 is an attractive alternative for researchers examining PA and sleep quality concurrently in older adults. However, there is an important gap in the literature, limiting the MW8's utility as a measure of PA. When used to evaluate sleep quality, the MW8 records data in $60 \mathrm{~s}$ epochs. The MW8 provides activity counts - summed in these $60 \mathrm{~s}$ epochs - but the problem is interpreting what these counts mean in terms of understanding PA. It makes intuitive sense that higher counts reflect a higher level of activity but what does that mean in terms of recommended levels of daily activity?

The current study aimed to fill this gap in the scientific literature. To achieve this objective we calibrated and validated cut-points for activity counts produced using the MW8 in healthy older adults. Cut-points are used to provide an estimate of activity level and intensity at which the activity is performed (Crouter et al., 2014). Typically, PA is categorized according to one of the following intensities: light, moderate, vigorous, or very-vigorous. Researchers then evaluate the number of minutes a participant engages in PA for a given intensity, over the duration of a specific observation period (e.g., 3 h, whole day; Trost, 2001). In addition, 
we aimed to determine the reliability of the MW8 for measuring PA in healthy older adults.

\section{Materials and Methods}

\section{Participants}

Community dwelling adults $55+(N=23)$ were recruited through advertising in newspapers, pamphlets distributed at local community centers, and word of mouth referrals. Individuals interested in participating in the study were pre-screened for eligibility criteria. We included individuals in the study who: (1) were cleared for exercise using the modified Physical Activity Readiness Questionnaire (Modified PAR-Q; Cardinal et al., 1996; Cardinal and Cardinal, 2000); (2) could walk independently with or without a walking aid; and (3) scored $\geq 24$ on the Mini-Mental State Exam (Folstein et al., 1975). We excluded participants who: (1) had a medical condition precluding them from exercise; (2) had a chronic respiratory condition that could be made worse by exercise; (3) were unable to wear a portable indirect calorimeter during testing; and (4) had limited mobility such that they could not walk $400 \mathrm{~m}$ independently. Participants were not excluded based on existence of common age-related co-morbidities such as type- 2 diabetes, hypertension, osteoporosis, or history of cancer. However, our inclusion/exclusion criteria were designed to ensure participants were healthy enough to engage in regular moderate to vigorous PA of their own volition. Note: we estimated a correlation between PA measures of $r \geq 0.5$, requiring a sample size $\geq 21$ to sufficiently power our statistical analyses $\left(G^{*}\right.$ Power 3.1; Faul et al., 2009).

Ethical approval for this study was obtained from the Vancouver Coastal Health Research Institute and the University of British Columbia's Clinical Research Ethics Board. All participants provided written informed consent.

\section{Measures \\ Wrist Worn Accelerometer}

We used the MW8 actigraphy system (CamNtech): a light weight, water-proof, tri-axial WWA. In accordance with standard protocol established in previous WWA validation studies, our participants wore the WWA on their non-dominant wrist (Crouter et al., 2014; Johansson et al., 2015). For the purposes of reliability testing, participants wore two WWAs on their nondominant wrist (Ekblom et al., 2012). Data from the distal WWA (MW8-1) were used to determine activity count cut-points for each PA intensity level (i.e., sedentary, light, and moderate to vigorous). Data from the proximal WWA (MW8-2) were used to test reliability. MW8 data were recorded using $60 \mathrm{~s}$ epochs, to match the recommended settings for sleep quality assessments.

\section{Indirect Calorimeter}

As our criterion measure we used the Cosmed k4b2, a portable indirect calorimeter (Cosmed; Rome, Italy) to determine energy expenditure (EE) during each assessment. Indirect calorimetry is used to measure oxygen uptake (VO2) and production of carbon dioxide (VCO2). Inspired and expired gasses are collected via a breathing mask and then analyzed to determine VO2 and VCO2 volumes. These measurements are used to determine EE in the form of metabolic equivalents (METs) via Schofield-equations (Schofield, 1985); which are then used to classify an activity based on the METs it requires. In accordance with established guidelines (Pate et al., 2008), activities were classified as follows: (1) sedentary (<1.5 METs); (2) light (1.5-3.0 METs), or moderate to vigorous physical activity (MVPA; $>3.0 \mathrm{METs})$.

\section{Demographics}

Participants were surveyed via questionnaire for their age, sex, race, and ethnicity; as well as any current or previous health conditions such as diabetes, hypertension, osteoporosis, arthritis, and cancer. In addition, participants reported if they used a cane or other ambulatory device. Finally, height and body weight (as measured by a calibrated stadiometer and electronic scale, respectively) were used to determine each participant's body mass index (BMI; kg/m2).

\section{Measurement Protocol}

The Cosmed gas analyzers were calibrated and verified with known gasses immediately before and after each test. Following calibration completion, the Cosmed was fitted for comfort to each participant. The Cosmed was worn concurrently with both WWAs (i.e., MW8-1 and MW8-2). To improve the accuracy of indirect calorimetry - in accordance with standard procedures (Bouten et al., 1994; Nichols et al., 2000; Schmitz et al., 2005) - participants were asked to fast $3 \mathrm{~h}$ prior to arriving for the measurement session.

We used $60 \mathrm{~s}$ epochs for WWA data collection, in accordance with previous validation studies (Berntsen et al., 2010). Measurement of METs was also recorded at $60 \mathrm{~s}$ intervals on the indirect calorimeter. Total EE (in kilocalories) during each session was determined using indirect calorimetry. The WWA, METs, and EE data from each session were downloaded into Microsoft Excel for further processing. To ensure consistency across measurement sessions, the same assessor - who was previously trained on all aspects of the measurement protocol - calibrated the Cosmed and conducted the trials for all participants. All instruments were synchronized to the same clock, and time was recorded at the beginning and end of each activity to ensure appropriate data comparisons could be made across recording devices. For all sessions, notes on participant activity during the trial were documented.

The measurement session lasted $\sim 60$ min, during which each participant performed 6 different activities designed to mimic activities of daily living: (1) treadmill walking at four different paces; (2) sitting in a chair; (3) cleaning; (4) resistance training; (5) lying down; and (6) standing. To more closely mimic freeliving activity, participants were allowed to move their arms freely during these activities. In order to minimize distraction and standardize the testing procedure the measurement session occurred in a quiet room and participants were not permitted to listen to music. Participants were provided standardized instructions (as per a predetermined script), prior to beginning each activity. As described below, participants performed 10 activities - for $5 \mathrm{~min}$ each - in the following order. 


\section{Treadmill Walking}

Participants were asked to walk at four different speeds on the treadmill, with each speed intended to mimic a specific pace: Leisurely, Comfortable, Moderate, and Brisk. Participants selfselected the treadmill speed for each pace, as described below. This was done for safety reasons, as well as to adjust for variability in fitness level across participants. Once the treadmill speed had been selected for each pace, the participant walked at this pace for $5 \mathrm{~min}$. The following order was used for each self-selected pace.

\section{Leisurely pace}

Participants were first instructed to walk at a leisurely pace. This treadmill speed was described as a pace the participant would walk at during a casual walk with a friend. Participants were instructed this pace was supposed to be easy, requiring minimal exertion.

\section{Comfortable pace}

Participants were instructed to walk at a pace they would use for a little light exercise. This treadmill speed was described as being faster than the previous speed, but requiring limited exertion.

\section{Moderate pace}

Participants were instructed to walk at a pace they would use for moderate exercise or when completing an errand. Participants were instructed to set the treadmill speed at a quick pace, so they would walk with purpose but not urgency.

\section{Brisk pace}

This treadmill speed was described as a pace the participant would walk if they were running late or needed to get somewhere as fast as possible. It was intended to be the fastest pace the participant could walk at for 5 min without slowing down.

\section{Sitting}

Participants were instructed to sit in a chair. No instructions were given as to how they must sit, but they were asked to remain seated for the entire $5 \mathrm{~min}$. Typically, the experimenter and participant engaged in polite conversation during this time.

\section{Cleaning}

Participants were given a broom and duster and asked to sweep the floor of the surrounding area. Participants were told to sweep the area as they normally would in their own home. The same marked off area was used for sweeping by each participant.

\section{Resistance Training}

Participants engaged in two resistance training activities that are typical for resistance training programs: Bicep Curls and Squats. Participants completed three sets of 10 repetitions for each exercise. Rest between sets of each exercise was permitted as needed.

\section{Bicep curls}

Instructions were provided as needed to ensure participants performed all bicep curls using proper technique in a slow and controlled manner while sitting. Five pound dumbbells and ten pound dumbbells were used by women and men, respectively.

\section{Squats}

Participants performed squats as chair squats. This required participants to sit down in a chair and then stand up to complete one repetition. Participants did not use weights for this task.

\section{Lying Down}

Participants were instructed to lie down on a bed for a period of 5 min.

\section{Standing}

Participants were asked to remain standing for $5 \mathrm{~min}$, but were given the option to move around the room freely. No additional instructions were given during this time as this period was intended to mimic standing and free-movement as part of routine daily activity. Following completion of this activity, the trial was terminated.

\section{Statistical Analysis Primary objective}

To derive MW8 activity count cut-points for healthy older adult PA levels for sedentary, light, moderate-to-vigorous activity: receiver operator characteristics (ROCs) analyses (Berk, 1976) were performed to determine optimal cut-points for the following PA intensities: sedentary activity ( $<1.5 \mathrm{METs})$, light activity (1.5-3.0 METs); MVPA (>3.0 METs; NIH, 2008; Pate et al., 2008). For each ROC curve, METs were coded as 0 or 1 according to the cut-point being established. For example, when the sedentary cutpoint was being established, a "1" was assigned to all minutes wherein a participant was reported in sedentary activity via indirect calorimetry and a " 0 " was assigned to all minutes wherein a participant was not reported in sedentary activity. The area under the ROC curve was calculated for PA intensity for both sedentary and MVPA.

Cut-points were established for sedentary activity using the point closest to $(0,1)$ on the ROC curve (i.e., $\left.d^{2}\right)$. This method of using the lowest $d^{2}$ is considered one of the two best methods for creating cut-points (Akobeng, 2007). Cut-points for MVPA were established using the lowest $d^{2}$ given a false-positive-ratio $<0.10$. We derived cut-points using this approach to prevent overestimation and provide a more conservative estimate of MVPA. The cut-point for light PA was determined as the activity level between the boundaries for sedentary activity and MVPA.

Sensitivity, Specificity, Accuracy, Positive Predictive Value, and Negative Predictive Value were calculated for both the sedentary activity and MVPA cut-points. Sensitivity is the percentage of epochs correctly identified as being engaged at the activity level examined (e.g., percent of actual MVPA epochs correctly identified as an epoch of MVPA). Specificity is the percentage of epochs correctly identified as not being engaged at the activity level examined (e.g., percent of actual non-MVPA epochs correctly identified as not being an epoch of MVPA). Accuracy is defined as the percent of correct decisions made using the derived cut-point (e.g., percent correctly identified as MVPA and percent correctly identified as non-MVPA). Positive Predictive Value is the probability of correctly identifying an epoch as being engaged at that activity level, given that it truly is an epoch of that activity level (e.g., probability of correctly identifying an epoch of MVPA 
as MVPA). Negative Predictive Value is the probability of correctly identifying an epoch as not being engaged at that activity level, given that it truly is not an epoch of that activity level (e.g., the probability of correctly identifying an epoch of sedentary activity as not being MVPA).

\section{Secondary objective}

To test reliability of the MW8: Using SPSS version 22 (IBM Corporation 2013), data for each WWA (i.e., MW8-1 and MW8-

2) were summarized and compared via interclass correlations (ICC) to assess average agreement for both WWA over different levels of activity. A higher ICC is indicative of greater inter-rater reliability. In addition, Bland-Altman plots were generated to determine agreement for both WWA across different levels of activity (Bland and Altman, 1986).

\section{Results}

\section{Participant Characteristics}

Table 1 describes characteristics of our study participants. The average age was 69.96 years $(\mathrm{SD}=6.57)$. Participants were slightly overweight (BMI: average $=26.64 \pm 5.22 \mathrm{~kg} / \mathrm{m} 2)$ as per current guidelines (Flegal et al., 2012) and were predominantly Caucasian (91.30\%) and female (69.60\%). In addition, participants had few self-reported comorbidities. Osteoporosis was the most common physical ailment reported (30.60\%), 4.30\% of participants used a cane or other ambulatory device.

\section{Reliability of WWA}

Table 2 describes the reliability of the WWA during the measurement period. A total of 1217 epochs were captured by

\begin{tabular}{|c|c|c|c|c|}
\hline \multicolumn{4}{|c|}{ Participant characteristic } & Mean (SD) or \% \\
\hline \multicolumn{4}{|l|}{ Age } & $69.96(6.57)$ \\
\hline \multicolumn{4}{|l|}{$\mathrm{BMI}$} & $26.64(5.22)$ \\
\hline \multicolumn{4}{|l|}{ Females } & $69.60 \%$ \\
\hline \multicolumn{5}{|l|}{ Race } \\
\hline \multicolumn{4}{|c|}{ Caucasian } & $91.30 \%$ \\
\hline \multicolumn{4}{|l|}{ Asian } & $4.30 \%$ \\
\hline \multicolumn{4}{|l|}{ Other } & $4.30 \%$ \\
\hline \multicolumn{4}{|l|}{ Diabetic } & $4.30 \%$ \\
\hline \multicolumn{4}{|l|}{ Cancer } & $4.30 \%$ \\
\hline \multicolumn{4}{|c|}{ Osteoporosis } & $30.40 \%$ \\
\hline \multicolumn{4}{|l|}{ Cane use } & $4.30 \%$ \\
\hline \multicolumn{5}{|c|}{ TABLE 2 | Reliability of the WWA. } \\
\hline Watch & $N$ & Mean CPM (SD) & $\begin{array}{l}\text { Pearson } \\
\text { correlation }\end{array}$ & ICC (95\% CI) \\
\hline Watch 1 & 1217 & $321.44(316.52)$ & $0.981 *$ & $\begin{array}{l}0.979(0.977 \\
0.981)^{*}\end{array}$ \\
\hline Watch 2 & 1217 & $276.84(295.72)$ & - & - \\
\hline
\end{tabular}

${ }^{*} p<0.01$. each WWA. Mean counts per minute (CPM) for MW8-1 was $321.44(\mathrm{SD}=316.52 \mathrm{CPM})$, while $\mathrm{MW} 8-2$ had a mean CPM of 276.84 ( $\mathrm{SD}=295.72 \mathrm{CPM})$. The average difference between WWA was 45 CPM as shown in Figure 1.

The CPM measured for MW8-1 and MW8-2 were strongly correlated $(r=0.981 ; p<0.01)$. Moreover, the ICC assessing consistency of the measures provided for each WWA also showed very strong agreement of ICC $=0.979$ (95\% CI: 0.977 , 0.981).

\section{ROC Curve Analysis}

Figure 2 describes the ROC curves for sedentary activity and MVPA, respectively. The area under the curve (AUC) for the sedentary ROC curve was deemed moderately accurate at 0.81 (95\% CI: $0.78,0.85)$, in accordance with previous recommendations for these analyses (Akobeng, 2007). The AUC for MVPA was also moderately accurate at 0.79 (95\% CI: 0.76 , $0.82)$.

\section{Determining Optimal Cut-points}

Table 3 describes the optimum cut-points for sedentary activity and MVPA with light activity being between these boundaries. The cut-point for sedentary behavior determined using the lowest $d^{2}$ was $\leq 178.50 \mathrm{CPM}\left(d^{2}=0.14\right)$. Using this cut-point yielded a sensitivity of $78 \%$ and specificity of $70 \%$, with an accuracy of $71 \%$. This sedentary activity cut-point had a positive predictive value of $39 \%$ and a negative predictive value of $93 \%$. After adjustments to target a false-positive ratio of $\leq 0.10$, the derived cut-point for MVPA was $\geq 562.50 \mathrm{CPM}\left(d^{2}=0.37\right)$. This MVPA cutpoint yielded a sensitivity of $40 \%$ and specificity of $90 \%$, with an accuracy of $69 \%$. This MVPA cut-point had a positive predictive value of $77 \%$ and a negative predictive value of $60 \%$.

\section{Discussion}

Most objectively measured studies of PA in older adults have used either a pedometer or hip-accelerometer to measure PA (Murphy, 2009). Pedometers are well established tools for measuring stepcounts and have been validated for the prediction of EE (Crouter et al., 2003). Hip-accelerometers have also been validated in various populations (Vooijs et al., 2014; Lee et al., 2015), including older adults (Copeland and Esliger, 2009; Taylor et al., 2014).

However, there are important limitations to consider when using hip accelerometers in interventions targeting older adults. First, few cut-points have been calibrated specifically for older adults. Importantly, Gorman et al. (2014) reviewed current studies using hip-accelerometers with older adults, and found the most commonly used cut-points had not been calibrated for older adults, but rather for young adults. This practice of using tools that have not been specifically calibrated for the population in question likely results in misleading findings (Falck et al., 2015). Second, a lack of wearer compliance can be an issue for hip-accelerometers - resulting in missing-data - which can also impact findings. And finally, hip accelerometers - and most pedometers - have not been designed for continuous wear 24/7. 


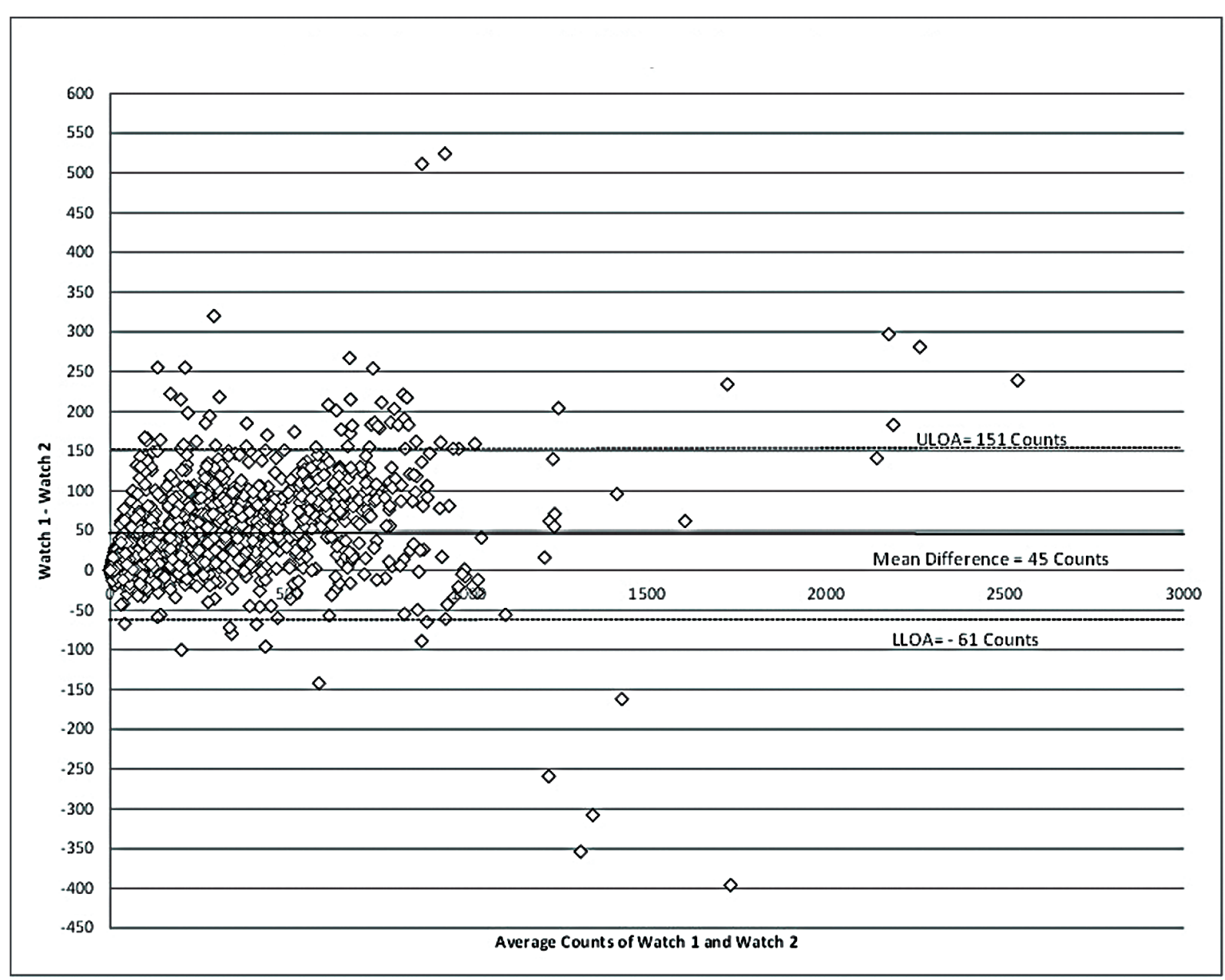

FIGURE 1 | Bland-Altman for reliability of the MotionWatch. Differences between counts reported by the distal watch (Watch 1) and proximal watch (Watch 2) are reported. A higher placement of the WWA on the wrist appears to be associated with a slight under-estimation of movement frequency. ULOA, upper limits of agreement; LLOA, lower limits of agreement.

By contrast, the MW8 was designed for long-term, continuous wear to record activity during the day and at night. The MW8 is the latest WWA, replacing the Actiwatch 4 and Actiwatch 7, which were discontinued. Importantly, the MW8 is waterresistant allowing for continuous wear, even during bathing, showers, and while swimming - providing an advantage over most pedometers and hip-accelerometers. Furthermore, the MW8 provides for both PA and sleep quality measurements in one device.

To our knowledge, we provide the first validation of PA cut-points for the MW8, specifically for use with healthy older adults 55+. Furthermore, our findings suggest that in addition to its previously validated use as a measure of sleep quality (Elbaz et al., 2012; Morgan et al., 2012) the MW8 is a reliable tool for measuring PA in healthy older adults. Considering the increasing number of studies testing interventions targeting PA and sleep quality and cognitive function in older adults (e.g., Ko and Youn, 2011; Nguyen and Kruse, 2012; Schega et al., 2013; Figueiro et al., 2014; Pa et al.,
2014; Richter et al., 2014; Cordi et al., 2015), and given the relationship between exercise and sleep (Driver and Taylor, 2000; Youngstedt, 2005), our PA cut-points validate a new level of analysis when using the MW8, filling an important gap in the literature.

\section{Limitations}

Our cut-points may be limited by inter-individual differences in physiological stress across participants. Numerous types of stressors including severe illnesses can affect EE (for review see Preiser et al., 2014); however, our sample was generally healthy - as evidenced by limited co-morbidities - such that minimal differences in basal metabolic rate would be expected. We acknowledge the MW8 activity count cut-points defined in the current study lack the accuracy previously reported for the GENEA accelerometer (Esliger et al., 2011). However, the MW8's utility as a valid objective measure of both PA and sleep quality, make it an attractive tool for researchers working with older adults. We expect the MW8 

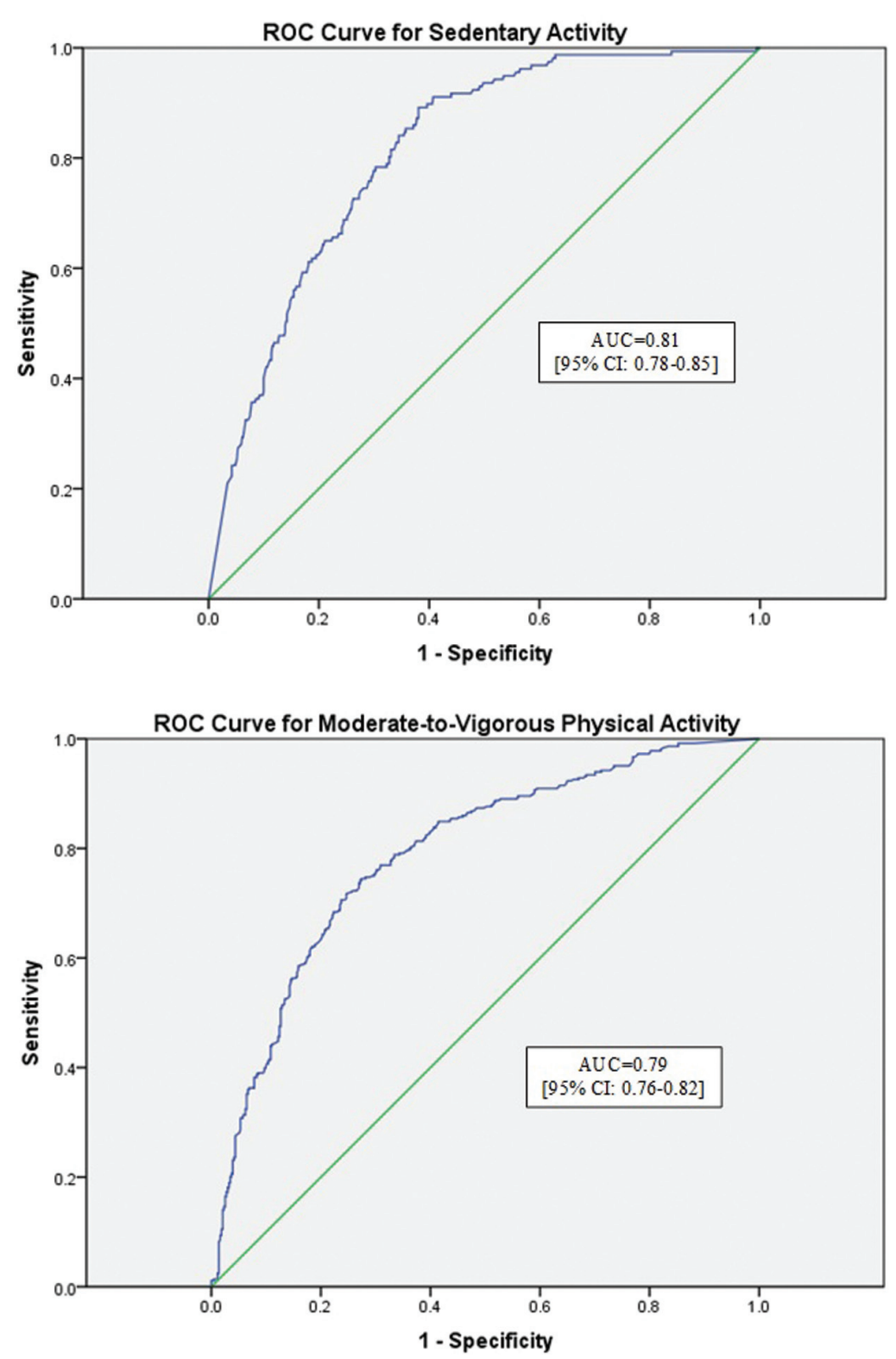

FIGURE 2 | Receiver operator characteristic (ROC) curves for sedentary activity and moderate-to-vigorous physical activity. ROC curves for both sedentary activity (Sensitivity $=0.78$; Specificity $=0.70$ ) and moderate-to-vigorous activity (Sensitivity $=0.34$; Specificity $=0.90)$ are reported. Moderate agreement was found for the WWA for both activity intensities. AUC, area under the curve.

TABLE 3 | Cut-points for Sedentary and Moderate-Vigorous Activity.

\begin{tabular}{|c|c|c|c|c|c|c|c|}
\hline Activity Level & Cut-point & Sensitivity & Specificity & Accuracy & $d^{2}$ & Positive predictive value & Negative predictive value \\
\hline Sedentary & $\leq 178.50$ & 0.78 & 0.70 & 0.70 & 0.22 & 0.39 & 0.93 \\
\hline MVPA* & $\geq 562.50$ & 0.34 & 0.90 & 0.67 & 0.37 & 0.77 & 0.60 \\
\hline Light** & $178.50-562.50$ & - & - & - & - & - & - \\
\hline
\end{tabular}

*Cut-point determined for lowest $d^{2}$ given specificity $\geq 0.90 .{ }^{* *}$ Cut-points for light activity are the boundaries for sedentary activity and MVPA.

to be used frequently in future studies, and thus, our cutpoints should provide for increased depth of analysis in these studies.
Generalizability of our findings is limited to MW8 use in healthy older adults. Older adults who are frail or confined to a wheelchair will likely have different PA patterns, requiring a 
unique set of PA cut-points. Furthermore, specific older adult populations such as those who suffer from chronic stroke would also require a unique set of PA cut-points.

\section{Acknowledgments}

Funding for this work was provided to TL-A by the Jack Brown and Family Alzheimer Research Foundation. GJL is a Canadian Institutes of Health Research PostDoctoral Fellow. TL-A is a Canada Research Chair Tier II

\section{References}

Ahlskog, J. E., Geda, Y. E., Graff-Radford, N. R., and Petersen, R. C.(2011). Physical exercise as a preventive or disease-modifying treatment of dementia and brain aging. Mayo Clin. Proc. 86, 876-884. doi: 10.4065/mcp.2011.0252

Akobeng, A. K. (2007). Understanding diagnostic tests 3: receiver operating characteristic curves. Acta Paediatr. 96, 644-647. doi: 10.1111/j.16512227.2006.00178.x

Ancoli-Israel, S., Cole, R., Alessi, C., Chambers, M., Moorcroft, W., and Pollak, C. P. $(2003)$. The role of actigraphy in the study of sleep and circadian rhythms. Sleep 26, 342-392.

Berk, R. A. (1976). Determination of optional cutting scores in criterion-referenced measurement. J. Exp. Educ. 45, 4-9. doi: 10.1080/00220973.1976.11011567

Berntsen, S., Hageberg, R., Aandstad, A., Mowinckel, P., Anderssen, S. A., Carlsen, K. H., et al. (2010). Validity of physical activity monitors in adults participating in free-living activities. Br. J. Sports Med. 44, 657-664. doi: 10.1136/bjsm.2008.048868

Blair, S. N. (2009). Physical inactivity: the biggest public health problem of the 21 st century. Br. J. Sports Med. 43, 1-2.

Blair, S. N., and Brodney, S. (1999). Effects of physical inactivity and obesity on morbidity and mortality: current evidence and research issues. Med. Sci. Sports Exerc. 31, S646-S662. doi: 10.1097/00005768-19991100100025

Bland, J. M., and Altman, D. G. (1986). Statistical methods for assessing agreement between two methods of clinical measurement. Lancet 1, 307-310. doi: 10.1016/S0140-6736(86)90837-8

Bouten, C. V., Westerterp, K. R., Verduin, M., and Janssen, J. D. (1994). Assessment of energy expenditure for physical activity using a triaxial accelerometer. Med. Sci. Sports Exerc. 26, 1516-1523. doi: 10.1249/00005768-19941200000016

Buysse, D. J., Reynolds, C. F. III, Monk, T. H., Berman, S. R., and Kupfer, D. J. (1989). The pittsburgh sleep quality index: a new instrument for psychiatric practice and research. Psychiatry Res. 28, 193-213. doi: 10.1016/01651781(89)90047-4

Cardinal, B. J., and Cardinal, M. K. (2000). Preparticipation physical activity screening within a racially diverse, older adult sample: comparison of the original and Revised Physical Activity Readiness Questionnaires. Res. Q. Exerc. Sport. 71, 302-307. doi: 10.1080/02701367.2000.10608910

Cardinal, B. J., Esters, J., and Cardinal, M. K. (1996). Evaluation of the revised physical activity readiness questionnaire in older adults. Med. Sci. Sports Exerc. 28, 468-472. doi: 10.1097/00005768-199604000-00011

Carney, C. E., Buysse, D. J., Ancoli-Israel, S., Edinger, J. D., Krystal, A. D., Lichstein, K. L., et al. (2012). The consensus sleep diary: standardizing prospective sleep self-monitoring. Sleep 35, 287-302. doi: 10.5665/sleep.1642

Chodzko-Zajko, W. J., Proctor, D. N., Fiatarone Singh, M. A., Minson, C. T., Nigg, C. R., Salem, G. J., et al. (2009). American college of sports medicine position stand. Exercise and physical activity for older adults. Med. Sci. Sports Exerc. 41, 1510-1530. doi: 10.1249/MSS.0b013e3181a0c95c

Colcombe, S., and Kramer, A. F. (2003). Fitness effects on the cognitive function of older adults: a meta-analytic study. Psychol. Sci. 14, 125-130. doi: 10.1111/14679280.t01-1-01430

Copeland, J. L., and Esliger, D. W. (2009). Accelerometer assessment of physical activity in active, healthy older adults. J. Aging Phys. Act. 17, 17-30. in Physical Activity, Mobility, and Cognitive Neuroscience. We would like to thank Dr. Jennifer Davis, who provided valuable suggestions during the preparation of the final manuscript.

\section{Supplementary Material}

The Supplementary Material for this article can be found online at: http://journal.frontiersin.org/article/10.3389/fnagi. 2015.00165

Cordi, M. J., Hirsiger, S., Merillat, S., and Rasch, B. (2015). Improving sleep and cognition by hypnotic suggestion in the elderly. Neuropsychologia 69, 176-182. doi: 10.1016/j.neuropsychologia.2015.02.001

Crouter, S. E., Flynn, J. I., and Bassett, D. R. Jr. (2014). Estimating physical activity in youth using a wrist accelerometer. Med. Sci. Sports Exerc. 47, 944-951. doi: 10.1249/MSS.0000000000000502

Crouter, S. E., Schneider, P. L., Karabulut, M., and Bassett, D. R. Jr. (2003). Validity of 10 electronic pedometers for measuring steps, distance, and energy cost. Med. Sci. Sports Exerc. 35, 1455-1460. doi: 10.1249/01.MSS.0000078932.61440.A2

Crowley, K. (2011). Sleep and sleep disorders in older adults. Neuropsychol. Rev. 21, 41-53. doi: 10.1007/s11065-010-9154-9156

Driver, H. S., and Taylor, S. R. (2000). Exercise and sleep. Sleep Med. Rev. 4, 387-402. doi: 10.1053/smrv.2000.0110

Ekblom, O., Nyberg, G., Bak, E. E., Ekelund, U., and Marcus, C. (2012). Validity and comparability of a wrist-worn accelerometer in children. J. Phys. Act. Health 9, 389-393.

Elbaz, M., Yauy, K., Metlaine, A., Martoni, M., and Leger, D. (2012). Validation of a New Actigraph Motion Watch Versus Polysomnography on 70 Healthy and Suspected Sleep-Disordered Subjects. (Hoboken, NJ: Journal of Sleep Research), 218-218.

Erickson, K. I., Weinstein, A. M., and Lopez, O. L. (2012). Physical activity, brain plasticity, and Alzheimer's disease. Arch. Med. Res. 43, 615-621. doi: 10.1016/j.arcmed.2012.09.008

Esliger, D. W., Rowlands, A. V., Hurst, T. L., Catt, M., Murray, P., and Eston, R. G. (2011). Validation of the GENEA accelerometer. Med. Sci. Sports Exerc 43, 1085-1093. doi: 10.1249/MSS.0b013e31820513be

Espiritu, J. R. (2008). Aging-related sleep changes. Clin. Geriatr. Med. 24, 1-14. doi: 10.1016/j.cger.2007.08.007

Falck, R. S., Mcdonald, S. M., Beets, M. W., Brazendale, K., and Liu-Ambrose, T. (2015). Measurement Falck of physical activity in older adult interventions: a systematic review. Br. J. Sports Med. doi: 10.1136/bjsports-2014-094413 [Epub ahead of print].

Faul, F., Erdfelder, E., Buchner, A., and Lang, A. G. (2009). Statistical power analyses using $\mathrm{G}^{*}$ Power 3.1: tests for correlation and regression analyses. Behav. Res. Methods 41, 1149-1160. doi: 10.3758/BRM.41.4.1149

Figueiro, M. G., Plitnick, B. A., Lok, A., Jones, G. E., Higgins, P., Hornick, T. R., et al. (2014). Tailored lighting intervention improves measures of sleep, depression, and agitation in persons with Alzheimer's disease and related dementia living in long-term care facilities. Clin. Interv. Aging 9, 1527-1537. doi: 10.2147/CIA.S68557

Flegal, K. M., Carroll, M. D., Kit, B. K., and Ogden, C. L. (2012). Prevalence of obesity and trends in the distribution of body mass index among US adults, 1999-2010. JAMA 307, 491-497. doi: 10.1001/jama.2012.39

Foley, D. J., Monjan, A. A., Brown, S. L., Simonsick, E. M., Wallace, R. B., and Blazer, D. G. (1995). Sleep complaints among elderly persons: an epidemiologic study of three communities. Sleep 18, 425-432.

Folstein, M. F., Folstein, S. E., and Mchugh, P. R. (1975). Mini-mental state A practical method for grading the cognitive state of patients for the clinician. J. Psychiatr. Res. 12, 189-198. doi: 10.1016/0022-3956(75)90026-6

Gorman, E., Hanson, H. M., Yang, P. H., Khan, K. M., Liu-Ambrose, T., and Ashe, M. C. (2014). Accelerometry analysis of physical activity and sedentary behavior in older adults: a systematic review and data analysis. Eur. Rev. Aging Phys. Act. 11, 35-49. doi: 10.1007/s11556-013-0132-x 
Hamer, M., and Chida, Y. (2009). Physical activity and risk of neurodegenerative disease: a systematic review of prospective evidence. Psychol. Med. 39, 3-11. doi: 10.1017/S0033291708003681

Hastings, M. H., Duffield, G. E., Smith, E. J., Maywood, E. S., and Ebling, F. J. (1998). Entrainment of the circadian system of mammals by nonphotic cues. Chronobiol. Int. 15, 425-445. doi: 10.3109/07420529808998700

Johansson, E., Ekelund, U., Nero, H., Marcus, C., and Hagstromer, M. (2015). Calibration and cross-validation of a wrist-worn Actigraph in young preschoolers. Pediatr. Obes. 10, 1-6. doi: 10.1111/j.2047-6310.2013.00213.x

Ko, H. J., and Youn, C. H. (2011). Effects of laughter therapy on depression, cognition and sleep among the community-dwelling elderly. Geriatr. Gerontol. Int. 11, 267-274. doi: 10.1111/j.1447-0594.2010.00680.x

Kowalski, K., Rhodes, R., Naylor, P. J., Tuokko, H., and Macdonald, S. (2012). Direct and indirect measurement of physical activity in older adults: a systematic review of the literature. Int. J. Behav. Nutr. Phys. Act. 9, 148. doi: 10.1186/1479-5868-9-148

Landry, G. J., Best, J. R., and Liu-Ambrose, T. (2015). Measuring sleep quality in older adults: a comparison using subjective and objective methods. Front. Aging Neurosci 7:166. doi: 10.3389/fnagi.2015.00166

Landry, G. J., and Liu-Ambrose, T. (2014). Buying time: a rationale for examining the use of circadian rhythm and sleep interventions to delay progression of mild cognitive impairment to Alzheimer's disease. Front. Aging Neurosci. 6:325. doi: 10.3389/fnagi.2014.00325

Lee, J. A., Williams, S. M., Brown, D. D., and Laurson, K. R. (2015). Concurrent validation of the Actigraph gt $3 \mathrm{x}+$, Polar Active accelerometer, Omron HJ720 and Yamax Digiwalker SW-701 pedometer step counts in lab-based and free-living settings. J. Sports Sci. 33, 991-1000. doi: 10.1080/02640414.2014.9 81848

Littner, M., Hirshkowitz, M., Kramer, M., Kapen, S., Anderson, W. M., Bailey, D., et al. (2003a). Practice parameters for using polysomnography to evaluate insomnia: an update. Sleep 26, 754-760.

Littner, M., Kushida, C. A., Anderson, W. M., Bailey, D., Berry, R. B., Davila, D. G., et al. (2003b). Practice parameters for the role of actigraphy in the study of sleep and circadian rhythms: an update for 2002. Sleep 26, 337-341.

Mistlberger, R. E., and Skene, D. J. (2004). Social influences on mammalian circadian rhythms: animal and human studies. Biol. Rev. Camb. Philos. Soc. 79, 533-556. doi: 10.1017/S1464793103006353

Mistlberger, R. E., and Skene, D. J. (2005). Nonphotic entrainment in humans? J. Biol. Rhythms. 20, 339-352. doi: 10.1177/0748730405277982

Morgan, P. L., Hampton, S., Karatziotou, A., Zaslona, J., and Middleton, B. (2012). Validation of two new activity monitors: motionwatch 8 and pro-diary motion. J. Sleep Res. 74, 217-218.

Mrosovsky, N. (1996). Locomotor activity and non-photic influences on circadian clocks. Biol. Rev. Camb. Philos. Soc. 71, 343-372. doi: 10.1111/j.1469185X.1996.tb01278.x

Murphy, S. L. (2009). Review of physical activity measurement using accelerometers in older adults: considerations for research design and conduct. Prev. Med. 48, 108-114. doi: 10.1016/j.ypmed.2008.12.001

Nelson, M. E., Rejeski, W. J., Blair, S. N., Duncan, P. W., Judge, J. O., King, A. C., et al. (2007). Physical activity and public health in older adults: recommendation from the American College of Sports Medicine and the American Heart Association. Med. Sci. Sports Exerc. 39, 1435-1445. doi: 10.1249/mss.0b013e3180616aa2

Nguyen, M. H., and Kruse, A. (2012). A randomized controlled trial of Tai chi for balance, sleep quality and cognitive performance in elderly Vietnamese. Clin. Interv. Aging 7, 185-190. doi: 10.2147/CIA.S32600

Nichols, J. F., Morgan, C. G., Chabot, L. E., Sallis, J. F., and Calfas, K. J. (2000). Assessment of physical activity with the Computer Science and Applications, Inc., accelerometer: laboratory versus field validation. Res. Q. Exerc. Sport. 71, 36-43. doi: 10.1080/02701367.2000.10608878

NIH. (2008). Physical Activity Guidelines for Americans. Bethseda, MD: National Institute of Health.
Pa, J., Goodson, W., Bloch, A., King, A. C., Yaffe, K., and Barnes, D. E. (2014). Effect of exercise and cognitive activity on self-reported sleep quality in communitydwelling older adults with cognitive complaints: a randomized controlled trial. J. Am. Geriatr. Soc. 62, 2319-2326. doi: 10.1111/jgs.13158

Pate, R. R., O'neill, J. R., and Lobelo, F. (2008). The evolving definition of sedentary. Exerc. Sport Sci. Rev. 36, 173-178. doi: 10.1097/JES.0b013e3181877d1a

Preiser, J. C., Ichai, C., Orban, J. C., and Groeneveld, A. B. (2014). Metabolic response to the stress of critical illness. Br. J. Anaesth. 113, 945-954. doi: 10.1093/bja/aeu187

Richter, K., Myllymaeki, J., Scharold-Schaefer, S., Tomova, I., Mayrer, R., and Niklewski, G. (2014). Treating comorbid insomnia in older adults via cognitivebehavioural treatment, bright light and exercise. Health (Irvine Calif.) 2014, 960-968. doi: 10.4236/health.2014.610121

Schega, L., Peter, B., Torpel, A., Mutschler, H., Isermann, B., and Hamacher, D. (2013). Effects of intermittent hypoxia on cognitive performance and quality of life in elderly adults: a pilot study. Gerontology 59, 316-323. doi: $10.1159 / 000350927$

Schmitz, K. H., Treuth, M., Hannan, P., Mcmurray, R., Ring, K. B., Catellier, D., et al. (2005). Predicting energy expenditure from accelerometry counts in adolescent girls. Med. Sci. Sports Exerc. 37, 155-161. doi: 10.1249/01.MSS.0000150084.97823.F7

Schofield, W. N. (1985). Predicting basal metabolic rate, new standards and review of previous work. Hum. Nutr. Clin. Nutr. 39(Suppl. 1), 5-41.

Swift, D. L., Lavie, C. J., Johannsen, N. M., Arena, R., Earnest, C. P., O’keefe, J. H., et al. (2013). Physical activity, cardiorespiratory fitness, and exercise training in primary and secondary coronary prevention. Circ. J. 77, 281-292. doi: 10.1253/circj.CJ-13-0007

Taylor, L. M., Klenk, J., Maney, A. J., Kerse, N., Macdonald, B. M., and Maddison, R. (2014). Validation of a body-worn accelerometer to measure activity patterns in octogenarians. Arch. Phys. Med. Rehabil. 95, 930-934. doi: 10.1016/j.apmr.2014.01.013

Trost, S. G. (2001). Objective measurement of physical activity in youth: current issues, future directions. Exerc. Sport Sci. Rev. 29, 32-36. doi: 10.1097/00003677200101000-00007

Vooijs, M., Alpay, L. L., Snoeck-Stroband, J. B., Beerthuizen, T., Siemonsma, P. C., Abbink, J. J., et al. (2014). Validity and usability of low-cost accelerometers for internet-based self-monitoring of physical activity in patients with chronic obstructive pulmonary disease. Interact. J. Med. Res. 3:e14. doi: 10.2196/ijmr.3056

Warren, J. M., Ekelund, U., Besson, H., Mezzani, A., Geladas, N., Vanhees, L., et al. (2010). Assessment of physical activity - a review of methodologies with reference to epidemiological research: a report of the exercise physiology section of the European Association of Cardiovascular Prevention and Rehabilitation. Eur. J. Cardiovasc. Prev. Rehabil. 17, 127-139. doi: 10.1097/HJR.0b013e32832ed875

WHO, and ADI. (2012). Dementia: A Public Health Priority. Geneva: World Health Organization.

Wimo, A., and Prince, M. J. (2010). World Alzheimer Report 2010: the Global Economic Impact of Dementia. London: Alzheimer's Disease International.

Youngstedt, S. D. (2005). Effects of exercise on sleep. Clin. Sports Med. 24, 355-365. doi: $10.1016 /$ j.csm.2004.12.003

Conflict of Interest Statement: The authors declare that the research was conducted in the absence of any commercial or financial relationships that could be construed as a potential conflict of interest.

Copyright (c) 2015 Landry, Falck, Beets and Liu-Ambrose. This is an open-access article distributed under the terms of the Creative Commons Attribution License (CC BY). The use, distribution or reproduction in other forums is permitted, provided the original author(s) or licensor are credited and that the original publication in this journal is cited, in accordance with accepted academic practice. No use, distribution or reproduction is permitted which does not comply with these terms. 